\title{
Efficient Regeneration from Hypocotyl Cultures of Betalain Forming Plant, Portulaca sp. cv. 'Jewel': Stimulatory Effect of Thidiazuron
}

\author{
Md. Nazmul Hossain BHUIYAN and Taiji ADACHI* \\ Graduate School of Agriculture and Biological Sciences, Osaka Prefecture University, \\ 1-1 Gakuen-cho, Sakai, Osaka, 599-8531, Japan \\ *Corresponding author E-mail address: taijiada@plant.osakafu-u.ac.jp
}

Received 23 April 2001; accepted 22 November 2001

\begin{abstract}
An efficient and simple in vitro regeneration protocol has been successfully developed from three inbred lines of Portulaca sp. cv. 'Jewel' that contain a lot of betalain. The present report describes the effect of thidiazuron [ $\mathrm{N}-$ phenyl- $\mathrm{N}^{\prime}(-1,2,3$ - thidiazol-5-yl) urea] (TDZ) on in vitro shoot regeneration from intact hypocotyl explant of seedlings. The optimum level of TDZ supplementation to the culture medium was $5 \mu \mathrm{M}$ for a 3 week-induction period followed by subculture of hypocotylderived callus on MS basal medium (MSO). Hypocotyls excised from 7 day-old seedling gave significantly higher number of regenerated shoots than those from 14 or 21 day-old seedling. The efficacy of thidiazuron was compared with $\mathrm{N}^{6}$ - benzylaminopurine (BAP) and $\mathrm{N}^{6}$ - furfurylaminopurine (KIN) (purine based cytokinins) on in vitro organogenesis. TDZ was found to be more effective than BAP and KIN as an inductive signal of regeneration. TDZ- supplemented medium with indole-3acetic acid was not effective for the regenerative response. Transfer of the TDZ-stimulated shoots to growth regulator free MSO medium containing 3\% sucrose resulted in the rapid and prolific growth of plantlets. In vitro shoots were rooted after culture on half-strength MS medium without growth regulator or in the presence of 2.5 or $5.0 \mu \mathrm{M}$ NAA and successfully acclimatized in greenhouse condition. The procedure developed in this study may be useful toward improvement and development of betalain through genetic manipulation.
\end{abstract}

Keywords: hypocotyl-derived callus, hypocotyls, in vitro regeneration, Portulaca sp. cv. 'Jewel', TDZ.

\begin{abstract}
Abbreviations
TDZ, thidiazuron; BAP, $\mathrm{N}^{6}$ - benzylaminopurine; KIN, $\mathbf{N}^{6}$ - furfurylaminopurine; PGR, plant growth regulator; MSO, Murashige and Skoog basal medium.
\end{abstract}

Cytokinin-like plant growth regulators are mainly two types; one is synthetic phenyl urea derivative including $\mathbf{N}$-phenyl- $\mathbf{N}^{\prime}(-1,2,3$-thidiazol-5-yl) urea (thidiazuron, TDZ) and the other is a naturally occurring purine-based derivative including $\mathbf{N}^{6}$ benzylaminopurine (BAP). In many bioassay systems, these compounds have been shown to induce similar physiological responses viz. regulation of cell division, growth and differentiation of tissues and organs (Mok et al., 1982). More recently, TDZ has emerged as a highly effective bioregulant in tissue cultures of a diverse array of species ranging from herbaceous to tree plants (Murthy et al., 1998).

The genus Portulaca belongs to the family Portu- lacaceae, and comprises more than 100 species, which are distributed in tropical, subtropical and temperate areas of the world. Portulaca grandiflora comes from South America and is popular as an ornamental plant in many countries (von Poellnitz, 1934) for its various colored flowers (red, magenta, yellow, orange, white etc.). Petals, stem and other parts of this plant contain a lot of betalain, instead of anthocyanin, which is used as natural colorant in food industries, pharmaceuticals and cosmetics (Leathers et al., 1992). Recently, betalain has gain attraction because betanin has shown antifungal and antimicrobial activities (Delgado-Vargas et al., 2000). Quantitative and qualitative identification of betalain pigments and their distribution in the genus Portulaca were analyzed by HPLC (Adachi and Nakatsukasa, 1983). Diverse callus lines displaying different colors (Noda and Adachi, 2000b) and suspension culture for betacyanin production were reported (Noda and Adachi, 2000a) from Portulaca sp. 'Jewel'. Nevertheless, the biosynthetic pathway 
of betalain is not yet well determined. Therefore, biotechnological application such as genetic transformation system is essential to improve the knowledge of the genetics and biochemistry of betalain. On the other hand, improvement and development of betalain by genetic engineering are future aspects under controlled conditions. However, an effective regeneration protocol is prerequisite for gene manipulation. There are only a couple of reports on in vitro propagation of Portulaca grandiflora with limited success. Konar (1978) reported that $P$. grandiflora cell cultures showed relatively slow growth and organogenesis occurred infrequently. Rossi-Hassani and Zryd (1995) obtained about 10 shoots from hypocotyl culture by using BA combined with NAA. In the present report, we describe a rather novel approach for establishment of an efficient regeneration protocol through hypocotyl culture by using thidiazuron [ $\mathrm{N}-$ phenyl $-\mathbf{N}^{\prime}(-$ 1,2,3-thidiazol-5-yl)] (TDZ) from three inbred line of Portulaca sp. cv. 'Jewel'. Moreover, we compare here the potential role of TDZ with that of $\mathbf{N}^{6}$-benzylaminopurine (BAP) and $\mathbf{N}^{6}$-furfurylaminopurine (KIN); two most commonly used purine - based cytokinins.

Seeds of three near isogenic inbred lines of Portulaca sp. cv. Jewel (Adachi et al., 1985); JM, JR and JW expressing magenta, red and white color petal, respectively, were surface sterilized by immersion in $70 \%$ ethanol solution for $1 \mathrm{~min}$, followed by immersion in $1 \%$ sodium hypochlorite aqueous solution for $15 \mathrm{~min}$. Seeds were then washed with sterile distilled water for several times. To promote germination, seeds were soaked in $1 \%$ thiourea for $30 \mathrm{~min}$. Sterile seeds were germinated and maintained on agar-distilled water medium. Seeds-containing vessels $(150-\mathrm{ml}$ conical flask) were kept under $16 \mathrm{~h} \mathrm{light/day}\left(25 \mu \mathrm{mol} \mathrm{m}^{-2} \mathrm{~s}^{-1}\right)$ at $26{ }^{\circ} \mathrm{C}$ in the growth chamber. Seeds were germinated within 4 days. Intact hypocotyls from 7,14 and 21 daysold seedlings were excised by sterile scalpel. The hypocotyl explants were inoculated on MS (Murashige and Skoog, 1962) basal medium containing 30 $\mathrm{g}^{-1}$ sucrose, $3 \mathrm{~g} \mathrm{l}^{-1}$ gellan gum and various concentrations $(1,2.5,5,10,20,25 \mu \mathrm{M})$ of TDZ, BAP and KIN alone or in combination with 1 or $2 \mu \mathrm{M}$ IAA in a series of experiments. For each plant growth regulator (PGR) treatment, 18 vessels each containing 5 explants were made. For determination of the optimal duration of exposure of the explants to the medium containing TDZ, BAP or KIN, 3 vessels (5 explants per vessel) of each treatment were subcultured on plant growth regulator-free MS medium (MSO) 1, 2, 3 and 4 weeks after culture initiation. TDZ, BAP and $\mathrm{KIN}$-treated explants were transferred not only on MSO but also on IAA ( 1 and $2 \mu \mathrm{M}$ ) and NAA ( 1 and $2 \mu \mathrm{M})$-supplemented media. In a separate experiment, the potentiality of carbon sources such as sucrose, glucose and fructose in different concentrations $(20,30,40$ and $50 \mathrm{mg} \mathrm{l}^{-1}$ ) were compared to maximize the shoot growth on MSO. The $\mathrm{pH}$ of all the culture media was adjusted to 5.7 before autoclaving at 121 ${ }^{\circ} \mathrm{C}$ for $20 \mathrm{~min}$. Cultures were maintained under $16 \mathrm{~h}$ light/day $\left(25 \mu \mathrm{mol} \mathrm{m}^{-2} \mathrm{~s}^{-1}\right)$ at $26^{\circ} \mathrm{C}$ in the growth chamber. For root initiation, isolated shoots (5-10 $\mathrm{cm}$ ) were cultured on medium containing halfstrength of MS salts and full strength of basal organic constituents without any plant growth regulators or with 2.5 or $5 \mu \mathrm{M}$ NAA. Cultures were maintained at $26^{\circ} \mathrm{C}$ under $16 \mathrm{~h} \mathrm{light/day.} \mathrm{Rooted}$ plantlets were removed from culture medium, rinsed in water and transferred into greenhouse condition for acclimatization.

The response of explants to TDZ depends on the concentration and the duration of exposure. Initially, non-organized tissues developed around the cut edges of explants on any concentration of TDZ and leaf-like multiple adventitious buds emerged directly from those non-organized tissues (Fig. 1A)

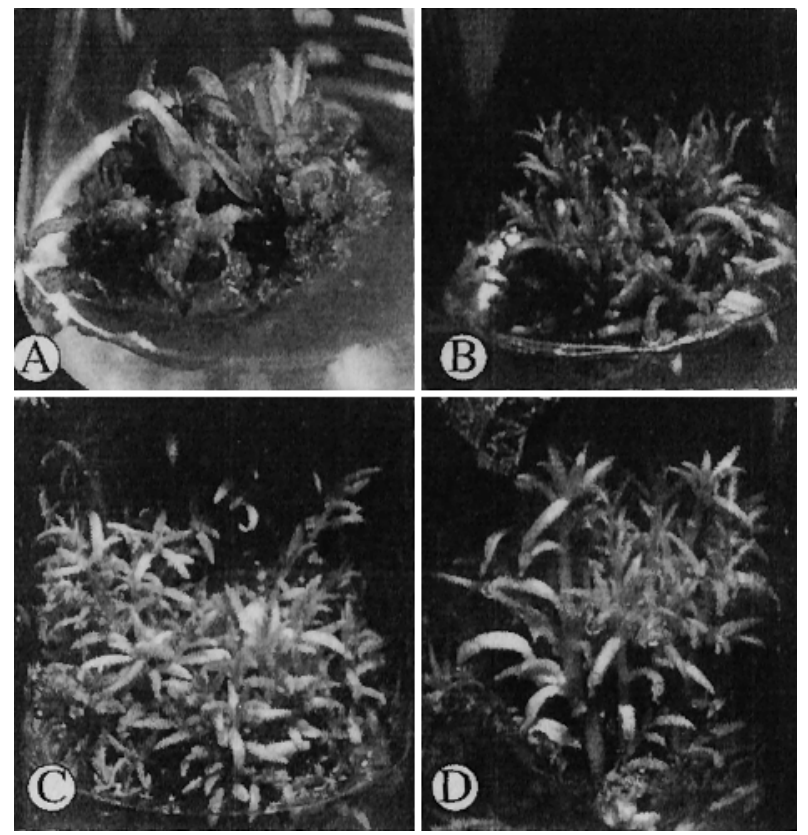

Fig. 1 Regeneration of shoots from hypocotyl culture of Portulaca sp. 'Jewel' JR. Hypocotyl explant was cultured on medium supplemented with TDZ $(5 \mu \mathrm{M})$ for 3 weeks and then hypocotyl-derived callus was subcultured on MSO. (A) Shoot primordia formation with red pigmentation from hypocotyl-derived callus 5 days after transfer onto MSO. (B) Multiple shoots 2 weeks after transfer onto MSO. (C) Proliferation of shoots after 3 weeks. (D) Elongation of shoots on MSO containing $3 \%$ sucrose. 


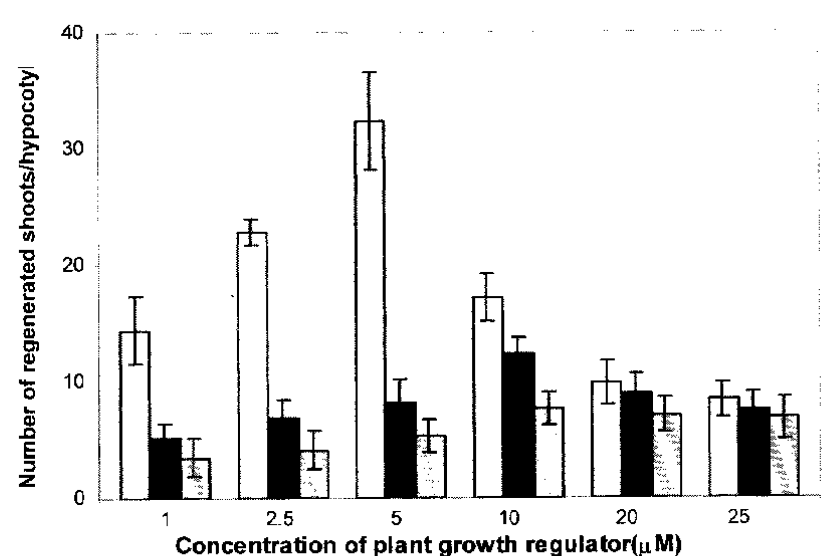

Fig. 2 A comparison of the efficacy of TDZ, BAP and KIN for induction of shoot organogenesis from hypocotyl culture of Portulaca sp. 'Jewel' JR (hypocotyls from 7 day-old seedling). White bar, black bar and gray bar represent TDZ, BAP and KIN, respectively. Data are presented in mean \pm standard deviation $(n=15)$.

with red pigmentation of betalain (only in JR \& JM lines). Subsequently these buds converted into complete shoots after transfer of the hypocotyl explants onto MSO (Fig. 1B, C, D). When the explant was cultured for 3 weeks or more in the presence of higher TDZ concentrations (5-25 $\mu \mathrm{M})$, the nonorganized tissues expanded, and the whole explant turned into a mass of tissue, which was defined as callus. All the calli were compact and greenish in color. Although hypocotyl formed larger callus in higher TDZ concentration, callus size did not have any role to increase the number of shoots formed.

TDZ-exposed hypocotyl or hypocotyl-derived callus produced multiple shoots within 3-4 weeks after transfer onto MSO or $1 \mu \mathrm{M}$ IAA or NAAsupplemented medium. In every treatment, hypocotyls or hypocotyl-derived calli produced significantly higher number of shoots on MSO in comparison with IAA or NAA-containing medium. Number of shoot formed per hypocotyl or callus depends on two important factors; one was the period of exposure to TDZ, and the other was the concentration of TDZ. The hypocotyl-derived callus, which had been formed by the treatment of 5 $\mu \mathrm{M}$ TDZ for 3 weeks, produced a mean of 32.4 shoots that was the highest number in our culture system, after transfer onto MSO. Explants, which remained on the medium containing higher concentration of TDZ for more than 3 weeks, induced less number of shoots via calli, and some of them showed browning symptoms and stunted growth. Moreover, some calli lost the ability to regenerate shoot.

The hypocotyls cultured on BAP or KIN $(1,2.5$, $5,10,20,25 \mu \mathrm{M})$-supplemented medium produced

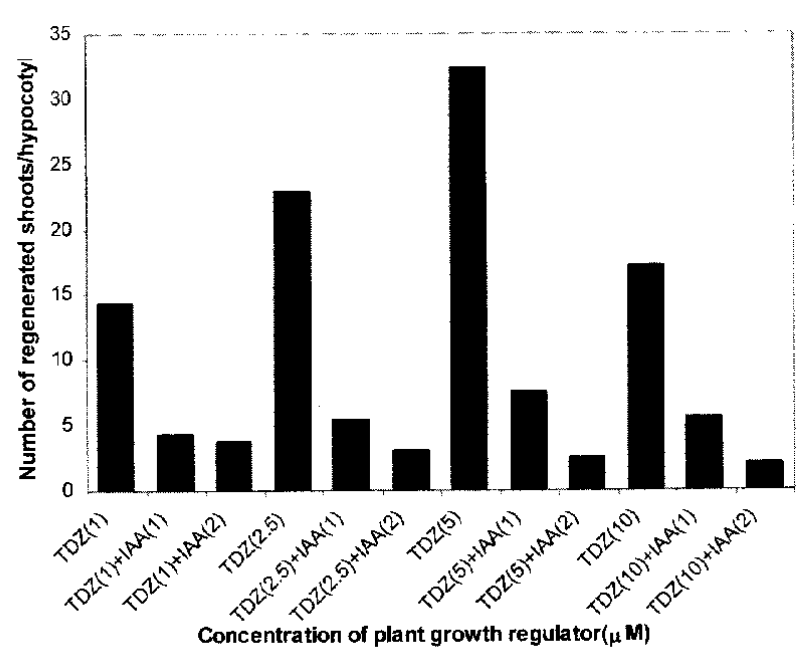

Fig. 3 Effects of TDZ and IAA on the induction of shoots on hypocotyl explants of Portulaca sp. 'Jewel' JR (hypocotyls from 7 day-old seedling). Data are presented in mean value $(n=15)$.

significantly less number of shoots than those treated with equimolar concentrations of TDZ or hypocotyl-derived calli as shown in Fig. 2. To invoke shoot organogenesis on BAP or KIN-treated hypocotyls, a continuous culture period (3-4 weeks) was needed, and one or two week-treatment failed to stimulate shoot regeneration after subculture on MSO. BAP stimulated to produce more shoots from hypocotyl than KIN. Among the 6 concentrations tested, $10 \mu \mathrm{M}$ of both purine-based cytokinins (BAP and KIN) gave the highest number of shoots (Fig. 2).

By adding 1 or $2 \mu \mathrm{M}$ IAA to the different concentration of TDZ-supplemented medium, hypocotyl explants produced compact light greenish callus within 2 weeks. In these calli, shoot number significantly reduced than those induced by TDZ alone after subcultured on MSO (Fig. 3). Similar response was observed when IAA was added with BAP or KIN-containing medium.

The effectiveness of TDZ on shoot regeneration was also observed in JM and JW inbred lines (Table 1). Hypocotyls exposed to $5 \mu \mathrm{M}$ TDZ for 3 weeks formed calli and subsequently those calli induced 25.2 regenerants in JM and 24.0 regenerants in JW lines, respectively after subculture on MSO. The induction of shoots per hypocotyl or hypocotylderived callus was also affected by seedling age. Hypocotyl excised from 7 day-old seedling significantly influenced the number of regenerated shoots per hypocotyl-derived callus than 14 or 21 day-old seedling. Three inbreed lines (JR, JM, JW) showed the same response. Among the three inbred lines, JR invoked highest number of shoots than JM and JW. Although shoot formation from intact seedling and 
Table 1 Effects of TDZ and seedling age on the number of shoots regenerated from hypocotyl culture of three inbred lines of Portulaca sp. 'Jewel'.

\begin{tabular}{cccc}
\hline \multirow{2}{*}{ Inbred line } & \multicolumn{3}{c}{ Seedling age (days) } \\
\cline { 2 - 4 } & 7 & 14 & 21 \\
\hline JR & $32.4 \pm 4.2$ & $24.2 \pm 1.8$ & $19.6 \pm 3.3$ \\
JM & $25.2 \pm 4.0$ & $20.2 \pm 2.5$ & $15.9 \pm 3.8$ \\
JW & $24.0 \pm 4.4$ & $19.4 \pm 2.6$ & $15.1 \pm 2.9$ \\
\hline
\end{tabular}

Hypocotyl explants were exposed with $5 \mu \mathrm{M}$ TDZ for 3 weeks and hypocotyl-derived calli were transferred onto MSO medium.

Note: Data are presented here in mean \pm standard deviation value $(n=15)$

petiole was also investigated by exposing TDZ, number of regenerants and frequency of regeneration were not so high as hypocotyl.

The cluster of shoots with the original explant was transferred to the fresh MSO for shoot elongation. Shoot elongation and growth depended on the type (sucrose, glucose and fructose) and concentration (2, 3, 4 and 5\%) of carbon sources. Best performance was observed in sucrose-containing medium than that of glucose and fructose and the optimum conc. was $3 \%$.

Elongated shoots were excised from the propagule mass and transferred to the rooting media which were full and half-strength MS basal salts with organic constituents without growth regulator or with $2.5 \mu \mathrm{M}$ or $5.0 \mu \mathrm{M}$ NAA. Half-strength MS gave better rooting response than full-strength MS. The frequencies of shoots with root formation after 5 weeks of culture were $37 \%$ on half-strength MS medium without PGR, 30\% with $2.5 \mu \mathrm{M}$ and $22 \%$ with $5.0 \mu \mathrm{M}$ NAA, respectively. The rooted plantlets were successfully transferred to the pots containing 1:1 mixture of soil and vermiculite under greenhouse condition with $90 \%$ survival.

The observation of the present study indicates that TDZ is the most potent growth regulator for the induction of shoots in Portulaca sp. 'Jewel'. Mean number of shoot formation per hypocotyl exposed to TDZ was comparatively higher than the previous report in Portulaca grandiflora, where BAP $(5 \mu \mathrm{M})$ and NAA $(2.5 \mu \mathrm{M})$ were used combindly (RossiHassani and Zryd, 1995). In the present study, exposure to lower TDZ conc. $(5 \mu \mathbf{M})$ for limited period of time ( 3 weeks) provided sufficient stimulus for optimum shoot induction without any other PGR combination. Interestingly, longer exposure to higher levels of TDZ resulted in residual effects, including a decrease in the number of regenerants and hyperhydricity of the shoot. Similar phenomenon was described previously (Lu, 1993; Murthy et al., 1998). In the present study, the stimulus for shoot organogenesis of TDZ was sufficient for shoot organogenesis from hypocotyl or hypocotylderived callus after subculture on MSO (growth regulator free medium). This indicates the capability of TDZ-stimulated hypocotyls or callus to synthesize required level of cytokinins or auxins for growth and proliferation after culture on PGR free medium. TDZ has been shown to induce the accumulation of both endogenous auxins and cytokinins in legumes and herbaceous species (Murthy et al., 1995; Hutchinson and Saxena, 1996).

The medium supplemented with auxin such as IAA or NAA produced less number of shoots than MSO from TDZ-treated hypocotyl or callus. In addition, TDZ-supplemented medium with IAA reduced the regenerative response (Fig. 3). From these results, we assumed that higher level of auxin was not suitable for regenerative response in Portulaca sp. Jewel.

In general, TDZ has been reported more effective than purine type cytokinins in shoot formation $(\mathrm{Lu}$, 1993; Murthy et al., 1998), which is consistent with our results; TDZ-treated hypocotyl or callus produced significantly higher regenerants than those treated with BAP or KIN. TDZ was successfully applied to induce shoot organogenesis in other recalcitrant species (Malik and Saxena, 1992; Akasaka et al., 2000).

Rooting response of TDZ-induced shoots was not satisfactory. Inadequate rooting on $\mathrm{TDZ}$-induced shoots was reported in previous studies ( $\mathrm{Lu}, 1993$; Khalafalla and Hattori, 1999). In earlier reports, evidence was shown that TDZ stimulate the ethylene level in closed culture system (Yip and Yang, 1986; Hutchinson et al., 1997) and that ethylene level inhibits the rooting in faba bean shoot culture (Khalafalla and Hattori, 2000). Defoliation, which occurred frequently in our culture system, supports the enhanced ethylene production within the vessel. Therefore, we predict that elevated ethylene level played some role to inhibit the root formation. Further experiment is necessary to increase the rooting percentage of $\mathrm{TDZ}$-stimulated in vitro shoots.

In conclusion, we have developed a simple and efficient procedure to regenerate Portulaca sp. 'Jewel' plants for the first time from hypocotyl or hypocotyl-derived callus of three inbreed lines (JR, $\mathrm{JM}, \mathrm{JW}$ ) by stimulatory effect of TDZ. This procedure will be utilized for gene manipulation in respect to enhance the betalain production and 
thereby facilitating the future development of betalain biosynthetic pathway research and genetic control.

\section{References}

Adachi, T., Nakatsukasa, M., 1983. High performance liquid chromatographic separation of betalains and their distribution in Portulaca grandiflora and related species. Z. Pflanzenphysiol., 109: 155-162.

Adachi, T., Nakatsukasa, M., Asaka, Y., Uta, T., 1985. Genetic analysis of some properties of flower color mutants found in the progenies of $\mathrm{X}$-ray irradiated Portulaca sp. 'Jewel'. Japan J. Breed, 35: 183-192.

Akasaka, Y., Daimon, H., Mii, M., 2000. Improved plant regeneration from cultured leaf in peanut (Arachis hypogea L.) by limited exposure to thidiazuron. Plant Sci., 156: 169- 175 .

Delgado-Vargas, F., Jimenez, A. R., Paredes-Lopez, O., 2000. Natural pigments: Carotenoids, anthocyanins, and betalains-Characteristics, biosynthesis, processing, and stability. Criti. Rev. Food Sci. and Nutri., 40: $173^{-}$ 289.

Hutchinson, J. M., Murr, D. P., Krishnaraj, S., 1997. Does ethylene play a role in thidiazuron-regulated somatic embryogenesis of geranium (Pelargonium $\mathrm{x}$ horturum) hypocotyl cultures. In Vitro Cell. Dev. Biol., 33: 136140.

Khalafalla, M. M., Hattori, K., 1999. A combination of thidiazuron and benzyladenine promotes multiple shoot production from cotyledonary node explants of faba bean (Vicia faba L.). Plant growth regulation, 27: 145148.

Khalafalla, M. M., Hattori, K., 2000. Ethylene inhibitors enhance in vitro root formation on faba bean shoots regenerated on medium containing thidiazuron. Plant growth regulation, 32: 59-63.

Konar, N. R., 1978. In vitro studies on Portulaca grandiflora Hook. Z. Pflanzenphysiol., 86: 443-451.

Leathers, R., Davin, C., Zryd, J. P., 1992. Betalain producing cell cultures of Beta vulgaris L.(red beet). In Vitro Cell. Dev. Biol., 28: $39-45$.

Lu, C. Y., 1993. The use of thidiazuron in tissue culture. In
Vitro Cell. Dev. Biol., 29: 92-96.

Malik, K. A., Saxena, P. K., 1992. Regeneration in Phaseolus vulgaris $\mathbf{L}$. High frequency induction of direct shoot formation in intact seedlings by $\mathbf{N}^{6}$ - benzyladenin and thidiazuron. Planta, 186: 384-389.

Mok, M. C., Mok, D. W. S., Armstrong, D. J., Shudo, K., Isogai, Y., Okanoto, T., 1982. Cytokinin activity of Nphenyl- $\mathbf{N}^{\prime}-1,2,3$-thidiazol-5-ylurea (thidiazuron). Phytochemistry, 21: 1509-1511.

Murashige, T., Skoog, F., 1962. A revised medium for rapid growth and bioassy with tobacco tissue culture. Physiol. Plant., 15: 473-497.

Murch, S. J., Choffe, K. L., Victor, J. M. R., Slimmon, T. Y., Krishnaraj, S., Saxena, P. K., 2000. Thidiazuron-induced plant regeneration from hypocotyl cultures of St. John's wort (Hypericum perforatum. cv 'Anthos'). Plant Cell Rep., 19: 576-581.

Murthy, B. N. S., Murch, S. J., Saxena, P. K., 1995. Thidiazuron induced somatic embryogenesis in intact seedlings of peanut (Arachis hypogea): Endogenous growth regulator levels and signifacance of cotyledons. Physiol. Plant., 94: 268-276.

Murthy, B. N. S., Murch, S. J., Saxena, P. K., 1998. Thidiazuron: a potent regulator of in vitro plant morphogenesis. In Vitro Cell. Div. Biol., 34: 267-275.

Noda, N., Adachi, T., 2000a. Induction of betacyanin synthesis and pigment accumulation in cell suspension cultures of Portulaca. Plant Biotechnol., 17: 27- 34.

Noda, N., Adachi, T., 2000b. Isolation of stable, variously colored callus lines in Portulaca sp. 'Jewel' and analysis of betalain composition. Plant Biotechnol., 17: $55^{-}$ 60.

Rossi-Hassani, B. D., Zryd, J. P., 1995. In Vitro culture and plant regeneration of large flower purslane. Plant Cell, Tissue Organ Cult., 41: 281 - 283.

Von Poellnitz, K., 1934. Versuch einer monographic der gattung Portulaca L. Feddes Repert, 34: 240-320.

Yip, W. K., Yang, S. F., 1986. Effect of thidiazuron, a cytokinin-active urea derivative, in cytokinin dependent ethylene production system. Plant Physiol., 80: 515 -519 . 